OPEN ACCESS

Edited by:

Francesco Doglietto,

University of Brescia, Italy

Reviewed by:

Jan Egger,

Graz University of Technology, Austria

Liverana Lauretti,

Università Cattolica del Sacro Cuore,

Italy

*Correspondence: Haijun Wang

wanghaij@mail.sysu.edu.cn

Yonghong Zhu

zhuyongh@mail.sysu.edu.cn

${ }^{\dagger}$ These authors have contributed equally to this work

Specialty section:

This article was submitted to

Pituitary Endocrinology,

a section of the journal

Frontiers in Endocrinology

Received: 28 July 2021 Accepted: 14 September 2021 Published: 30 September 2021

Citation:

Chen W, Wang M, Duan C, Yao S, Jiao $H$, Wang Z, Hu B, Mao Z, Zhu Y and Wang $H$ (2021) Prediction of the

Recurrence of Non-Functioning

Pituitary Adenomas Using

Preoperative Supra-Intra Sellar Volume and Tumor-Carotid Distance.

Front. Endocrinol. 12:748997.

doi: 10.3389/fendo.2021.748997

\section{Prediction of the Recurrence of Non-Functioning Pituitary Adenomas Using Preoperative Supra-Intra Sellar Volume and Tumor-Carotid Distance}

\author{
Wenli Chen ${ }^{1 \dagger}$, Mengqi Wang ${ }^{1 \dagger}$, Chengbin Duan ${ }^{1 \dagger}$, Shun Yao ${ }^{1}$, Haosen Jiao ${ }^{1}$, \\ Zongming Wang ${ }^{1}$, Bin $\mathrm{Hu}^{1}$, Zhigang Mao ${ }^{1}$, Yonghong $\mathrm{Zhu}^{2 *}$ and Haijun Wang ${ }^{1 *}$ \\ ${ }^{1}$ Center for Pituitary Surgery, Department of Neurosurgery, The First Affiliated Hospital, Sun Yat-sen University, \\ Guangzhou, China, ${ }^{2}$ Department of Histology and Embryology, Zhongshan School of Medicine, Sun Yat-Sen University, \\ Guangzhou, China
}

Background: Currently, it is difficult to estimate the possibility of recurrence of nonfunctioning pituitary adenomas (NFPAs). Markers such as Ki-67 or transcription factors rely on postoperative pathology, while few indices can be used for preoperative prediction. Therefore, we aimed to investigate the predictive effectiveness of supraintrasellar volume and tumor-carotid distance based on measurements derived from preoperative magnetic resonance imaging (MRI) data.

Method: Ninety-eight cases of NFPAs were evaluated, along with their clinical characteristics and MRI features. Four radiologic indices were analyzed, including intrasellar tumor volume, suprasellar tumor volume, maximum horizontal tumor diameter, and intercarotid distance. The ratio of supra-intrasellar volume and ratio of tumor-carotid distance were measured using 3D Slicer software, and the sum of two ratios was defined as the $\mathrm{V}$-D value. The correlation between recurrence and multiple factors was analyzed using univariate and multivariate logistic regression and KaplanMeier analysis, and ROC curves were used to estimate the prognostic performance of radiologic measurements in NFPAs.

Result: The supra-intrasellar volume ratio, tumor-carotid distance ratio and V-D value were significantly correlated with the recurrence of NFPAs. The predictive importance of the $\mathrm{V}$-D value reached $84.5 \%$, with a sensitivity of $83.7 \%$ and specificity of $67.3 \%$. The cutoff limit of the V-D value was 1.53, and patients with V-D values higher than 1.53 tended to relapse much earlier.

Conclusion: The V-D value has predictive importance for the recurrence of NFPAs preoperatively. Patients with higher $V$-D values will undergo recurrence earlier and should be given greater consideration in terms of surgery and follow-up time.

Keywords: non-functioning pituitary adenomas, V-D value, recurrence, prediction, pre-operative 


\section{INTRODUCTION}

Nonfunctioning pituitary adenomas (NFPAs), accounting for $15 \%-54 \%$ of all types of pituitary adenomas (PAs) (1), are characterized by a lack of hormonal oversecretion except for the high level of prolactin, as well as subclinical or silent hormone dysfunction caused by the "pituitary stalk effect" (2). Over $60 \%$ of NFPAs are macroadenomas (diameter over $10 \mathrm{~mm}$ ), and $57 \%$ are invasive (3). Most patients present with headaches, hormone disorders, visual impairment, or cranial nerve palsy. The diagnosis of most NFPAs is usually delayed by a mean time of $1.96 \pm 2.9$ years due to the absence of endocrine symptoms $(4,5)$. Complete surgical resection can only be achieved in approximately $40-50 \%$ of all patients, although surgery is still the first-line strategy in NFPAs (6). Over $10-20 \%$ of cases with gross tumor resection will experience relapse 5-10 years postoperatively. This number rises to $40 \%$ and $50 \%$ in 5 years and 10 years, respectively, if there is a residual tumor after operation (7).

However, there is a great degree of heterogeneity among cases in terms of tumor recurrence. According to the $2017 \mathrm{WHO}$ classification of brain tumors, most NFPAs are considered gonadotropin adenomas with a low chance of relapse. However, some subtypes of NFPAs, such as silent ACTH adenoma, Pit-1positive adenomas or plurihormonal adenomas, have a high risk of recurrence but can only be affirmed by postoperative pathological tests. The monoclonal antibody Ki-67 is often used as a tool to aid in recurrence prediction, but its accuracy is still controversial (1). Currently, there are few reliable indices for the prediction of the recurrence of NFPAs (8). The preoperative evaluation still relies on classic methods, including the Hardy-Wilson, and Knosp classifications. Some researchers have reported that these two systems are not effective for precise prediction of tumor recurrence (9-11). The Hardy-Wilson system mainly reflects the longitudinal extension of the tumor, while the Knosp system reflects horizontal invasiveness.

Invasion into the cavernous and/or sphenoid sinus has been proven to be effective in the prediction of recurrence in NFPAs (12) but quantitative analysis is necessary for precise evaluation. Yan et al., have already used the intercarotid distance for the prediction of total resection of PAs, which is closely related to recurrence (13). Therefore, we aim to develop a brief and effective evaluation system using intercarotid distance and supra-intrasellar volume for quantifying the horizontal and longitudinal extension of the tumor. Although many predictive models have already been built, our study aims to provide an evaluation system that is easy to manipulate and use routinely.

\section{MATERIALS AND METHODS}

\section{Patient Samples}

A total of 206 patients diagnosed with NFPAs by preoperative pituitary hormones, clinical behavior and postoperative immunohistochemistry were included in this study. After removal of the cases without adequate clinical or radiologic data, 98 cases who had undergone endoscopic trans-sphenoidal surgery in the First Affiliated Hospital, Sun Yat-sen University (Guangzhou, China) between January 2012 and December 2019. were finally selected. According to the tumor size, NFPAs can be classified into small adenomas (tumor largest diameter $<1.5 \mathrm{~cm}$ ), large adenomas $(1.5<=$ tumor largest diameter $<3 \mathrm{~cm})$ and giant adenomas $(\geq 3 \mathrm{~cm})$. Knosp and Hardy-Wilson grades were also evaluated by 2 doctors of the neurosurgical department independently.

Hormone-secreting PAs, NFPAs combined with other tumors and recurrent NFPAs were excluded from this study. The laboratory examination of serum hormones including prolactin (PRL), growth hormone $(\mathrm{GH})$, insulin-like growth factor-1 (IGF-1), thyroid-stimulating hormone (TSH), luteinizing hormone (LH), and follicle-stimulating hormone (FSH) was performed on the first day of attendance. All kinds of functioning pituitary adenomas were excluded according to the hormone levels and pathologic results. To prevent the potential errors caused by the "hook effect", serial dilution of the samples and re-measuring was performed.

Tumor recurrence was defined as (1): a tumor larger than 0.1 $\mathrm{cm}^{3}$ after total resection or (2) a residual tumor increased more than $25 \%$ after subtotal resection, during follow-up. Recurrence time was defined as the duration from the time of the first surgery to the first time of positive radiological findings. This study was approved by the Ethics Committee of The First Affiliated Hospital of Sun Yat-Sen University and was done in accordance with the Declaration of Helsinki.

\section{Imaging Protocol}

All patients had undergone sellar MRI using a high-field magnetic system (3.0T, Magnetom Verio, Siemens Healthcare, Erlangen, Germany) with a 64-channel head/neck coil. The regular sequences included coronal and sagittal T1-weighted turbo spinecho (TSE) with and without contrast (TR $=550 \mathrm{~ms}, \mathrm{TE}=8 \mathrm{~ms}$, thickness/gap $=2 / 1 \mathrm{~mm})$ and coronal and sagittal T2-weighted TSE sequences $(\mathrm{TR}=4000 \mathrm{~ms}$, TE $=116 \mathrm{~ms}$, thickness $/$ gap $=$ $2 / 1 \mathrm{~mm})$

\section{Imaging Processing}

All the images were exported from the radiological department and transformed into the Neuroimaging Informatics Technology Initiative (NIFTI) format using Mango (v4.1) software, which is a standard format for image processing. The pituitary adenomas were delineated using 3D Slicer (v4.11.0), open-source software for medical image processing, on sagittal T1-weighted sequences with contrast and adjusted on coronal T1-weighted and T2-weighted sequences with contrast. Two experienced neurosurgeons independently contoured all tumors blindly with the semiautomated module in the software (14). A senior neurosurgical professor helped with the finalization of tumor segmentation if the discrepancy of tumor volume estimation was over $20 \%$ between the two neurosurgeons. The suprasellar and intrasellar parts of tumors were divided according to the diaphragm sella. If the sellar diaphragm was unclear due to the 
invasion of the tumor, the horizontal level of the "waist sign" was considered the boundary between the suprasellar part and the intrasellar part. The suprasellar volume (V1) and intrasellar volume (V2) were also calculated using 3D Slicer. The infrasellar parts of tumors, such parts invading into the sphenoid sinus and clivus were included in the intrasellar volume. The intercarotid distance was defined as the shortest distance between its inner surface on the layer in which the tumor had the longest horizontal diameter within the intrasellar part. Both the longest horizontal diameter of the tumor (D1) and the corresponding intercarotid distance (D2) were calculated using 3D Slicer. The V-D value was defined as the sum of two ratios, V1/V2 and D1/D2.

\section{Histological Analysis}

Tumor samples were collected after transsphenoidal surgery, and routine $\mathrm{H} \& \mathrm{E}$ and immunohistochemical staining were performed in the Pathology Department in our hospital.

The Ki-67 value was reported as the percentage of tumor cells with positive nuclei in the lesion areas with the greatest labeling density, known as hotspots, using high-power fields (HPF 0.30 $\mathrm{mm} 2$, 400x magnification).

\section{Statistical Analysis}

All data were analyzed using the $\mathrm{R}$ program (v4.0.3). The difference between the two groups was determined by Student's t-test if the data had a normal distribution or a nonparametric test if the data did not have a normal distribution. The relationship between V-D value and clinical characteristics was assessed by Spearman's correlation. The receiver operating curve (ROC) was constructed to estimate the predictive capacity of $\mathrm{V}-\mathrm{D}$ system, and the area under curve (AUC) was also calculated using the "pROC" package in the $\mathrm{R}$ program. The Kaplan-Meier method was applied to assess the differences in the recurrence-free interval between patients grouped by the cutoff value of V-D system using "survival" and "survminer" packages in R. A p value of less than 0.05 indicated statistical significance.

\section{RESULTS}

\section{General Characteristics}

There were 45 males and 53 females ranging from 14 to 79 years old included in this study. The clinical characteristics of the 98

TABLE 1 | Baseline information (data of normal distribution were shown as means (standard deviation), data that did not fit the normal distribution were shown as means (median, interquartile range).

\begin{tabular}{|c|c|c|c|}
\hline & Non-recurrent & Recurrent & $\mathbf{p}$ \\
\hline $\mathrm{n}$ & 43 & 55 & \\
\hline Sex [Male(\%)] & $24(55.8)$ & 21 (38.2) & 0.125 \\
\hline age [mean (SD)] & $46.42(14.53)$ & $43.60(12.59)$ & 0.307 \\
\hline Knosp grade (\%) & & & 0.034 \\
\hline 0 & $2(4.7)$ & $0(0.0)$ & \\
\hline 1 & $16(37.2)$ & $9(16.4)$ & \\
\hline 2 & 7 (16.3) & $15(27.3)$ & \\
\hline 3 & $12(27.9)$ & $18(32.7)$ & \\
\hline 4 & $6(13.9)$ & 13 (23.6) & \\
\hline Hardy grade (\%) & & & 0.279 \\
\hline । & $13(30.2)$ & $7(12.7)$ & \\
\hline$\|$ & $15(34.9)$ & $22(40.0)$ & \\
\hline III & 8 (18.6) & 17 (30.9) & \\
\hline IV & $6(14.0)$ & $8(14.5)$ & \\
\hline V & $1(2.3)$ & $1(1.8)$ & \\
\hline Tumor size (\%) & & & 0.012 \\
\hline Giant (D1>3) & 8 (18.6) & $20(36.4)$ & \\
\hline Large $(1.5<=\mathrm{D} 1 \leq 3)$ & $21(48.8)$ & $29(52.7)$ & \\
\hline Small (D1<1.5) & $14(32.6)$ & $6(10.9)$ & \\
\hline Total tumor volume [median (IQR)] & $2648.33[873.97,6911.56]$ & 7920.27 [5313.34, 6573.73] & $<0.001$ \\
\hline V2 $\left(\mathrm{mm}^{3}\right)$ [median (IQR)] & $1808.24[873.97,5768.55]$ & $4635.93[3330.06,8449.81]$ & 0.002 \\
\hline V1 $\left(\mathrm{mm}^{3}\right)$ [median (IQR)] & $0.00[0.00,1184.99]$ & $3085.51[804.66,7717.00]$ & $<0.001$ \\
\hline V1N2 [median (IQR)] & $0.00[0.00,0.28]$ & $0.67[0.22,1.29]$ & $<0.001$ \\
\hline \multicolumn{4}{|l|}{ Diameter } \\
\hline D1 (mm) [median (IQR)] & $22.90[18.05,29.85]$ & $30.30[24.35,35.80]$ & $<0.001$ \\
\hline D2 (mm) [median (IQR)] & $24.30[22.50,28.55]$ & $27.30[24.70,31.20]$ & 0.043 \\
\hline D1/D2 [median (IQR)]) & $0.87[0.76,1.08]$ & $1.05[0.92,1.27]$ & $<0.001$ \\
\hline V-D value [median (IQR)] & $1.02[0.76,1.40]$ & $1.88[1.38,2.41]$ & $<0.001$ \\
\hline Time of recurrence [median (IQR)] & - & $14.00[6.50,32.50]$ & - \\
\hline Ki-67 [median (IQR)] & $1.00[0.00,1.00]$ & $1.00[1.00,1.00]$ & 0.142 \\
\hline PRL (ng/ml) [median (IQR)] & $19.90[13.38,34.34]$ & $20.52[10.93,38.98]$ & 0.98 \\
\hline Testosterone (ng/ml) [median (IQR)] & $0.58[0.24,1.45]$ & $0.36[0.21,0.71]$ & 0.1 \\
\hline $\mathrm{LH}(\mathrm{ng} / \mathrm{ml})[$ median $(\mathrm{IQR})]$ & $1.94[1.22,3.40]$ & $1.48[0.70,3.04]$ & 0.097 \\
\hline FSH (ng/ml) [median (IQR)] & $4.13[2.45,5.92]$ & $4.23[2.47,6.36]$ & 0.728 \\
\hline
\end{tabular}


NFPA patients are presented in Table 1. All patients signed informed consent forms, and the study met with approval from the ethics committee for Clinical Research and Animal Trials of The First Affiliated Hospital of Sun Yat-Sen University (Guangzhou, China). There were no significant differences between the nonrecurrent group (NR) and recurrent group (RE) in terms of age, sex, Hardy-Wilson grade, Ki-67 index, or other hormone levels, including PRL, testosterone, LH, and FSH. Knosp grade, tumor size, volumes, and diameters were significantly different between the two groups.

\section{The Relation Between the VD System and Clinicopathological Features}

Neither the level of hormones nor the Ki-67 index was correlated with the V-D value (Table 2). However, there was a significant correlation between the $\mathrm{V}-\mathrm{D}$ value and recurrence time. Univariate logistic regression showed that the suprasellar volume, total tumor volume, supra-intrasellar ratio, Knosp grade, maximum tumor diameter, tumor-ICA ratio, and V-D value had a significant correlation with the recurrence of NFPAs, while the levels of Ki-67 and other hormones had no statistical correlation with recurrence (Table 3). Furthermore, multivariate logistic regression was performed, and the results show that the supra-intrasellar ratio, tumor-ICA ratio, and $\mathrm{V}-\mathrm{D}$ value significantly correlated with recurrence (Table 4).

\section{The Predictive Value of the VD System Recurrence Compared to the Hardy and Knosp Systems}

The predictive effectiveness of the $\mathrm{V}$-D value was evaluated using ROC analysis (Figure 1). The area under the curve (AUC) reached $84.5 \%$, with a specificity of $67.3 \%$ and sensitivity of $83.7 \%$, showing that the predictive value was high compared to that of the Knosp and Hardy-Wilson grades. The cutoff limit of the $\mathrm{V}$-D value was 1.53 .

\section{The Relation Between the VD System and Recurrence Time}

We further subdivided the recurrent group into a large group (V-D >1.53) and a small group (V-D <1.53) using the cutoff value of $\mathrm{V}$-D. The survival curve of the two groups showed that the large group had a shorter recurrence time than the small group (Figure 2). Therefore, the V-D value can also reflect the probability of early recurrence in those patients (Figures 3, 4).

\section{DISCUSSION}

NFPAs are benign intracranial tumors arising from adenohypophyseal cells. Patients usually visit the clinic much later than the time of disease onset, with symptoms caused by the

TABLE 2 | Correlation between V-D value, Ki-67 and other hormone levels.

\begin{tabular}{|c|c|c|c|c|c|c|}
\hline Characteristic & V-D value & Ki-67 & PRL (ng/ml) & Testosterone (ng/ml) & LH (ng/ml) & FSH (ng/ml) \\
\hline V-D value & 1 & 0.16751 & 0.152164 & 0.090707 & -0.07162 & 0.211216 \\
\hline Ki-67 & 0.16751 & 1 & -0.26028 & 0.068129 & -0.1286 & -0.04428 \\
\hline $\mathrm{PRL}(\mathrm{ng} / \mathrm{ml})$ & 0.152164 & -0.26028 & 1 & -0.01206 & 0.003982 & 0.164758 \\
\hline Testosterone (ng/ml) & 0.090707 & 0.068129 & -0.01206 & 1 & 0.071892 & 0.054833 \\
\hline $\mathrm{LH}(\mathrm{ng} / \mathrm{ml})$ & -0.07162 & -0.1286 & 0.003982 & 0.071892 & 1 & 0.612763 \\
\hline FSH (ng/ml) & 0.211216 & -0.04428 & 0.164758 & 0.054833 & 0.612763 & 1 \\
\hline
\end{tabular}

TABLE 3 | Univariate Logistic regression between recurrence and multiple factors.

\begin{tabular}{|c|c|c|c|c|}
\hline & Coef & S.E. & Wald & $\mathbf{P}$ \\
\hline $\mathrm{V} 2\left(\mathrm{~mm}^{3}\right)$ & 0.0002 & 0.0001 & 1.10 & 0.2692 \\
\hline $\mathrm{V} 1\left(\mathrm{~mm}^{3}\right)$ & 0.0005 & 0.0001 & 3.70 & 0.0002 \\
\hline Total tumor volume $\left(\mathrm{mm}^{3}\right)$ & 0.0001 & 0.0000 & 3.23 & 0.0012 \\
\hline V1N2 & 2.6700 & 0.6537 & 4.08 & $<0.0001$ \\
\hline Knosp grade & 0.4133 & 0.1879 & 2.20 & 0.0278 \\
\hline Hardy grade & 0.9556 & 0.9673 & 0.99 & 0.3232 \\
\hline Tumor size & 2.5614 & 1.4856 & 1.72 & 0.0847 \\
\hline D1 (mm) & 0.0973 & 0.0281 & 3.46 & 0.0005 \\
\hline $\mathrm{D} 2(\mathrm{~mm})$ & 0.0714 & 0.0413 & 1.73 & 0.0842 \\
\hline D1/D2 & 2.6388 & 0.8612 & 3.06 & 0.0022 \\
\hline $\mathrm{Ki}-67$ & 0.4325 & 0.2709 & 1.60 & 0.1103 \\
\hline $\mathrm{PRL}(\mathrm{ng} / \mathrm{ml})$ & 0.0040 & 0.0090 & 0.44 & 0.6574 \\
\hline $\mathrm{LH}(\mathrm{ng} / \mathrm{ml})$ & -0.0550 & 0.0677 & -0.81 & 0.4160 \\
\hline FSH (ng/ml) & 0.0039 & 0.0255 & 0.15 & 0.8769 \\
\hline Testosterone (ng/ml) & -0.1255 & 0.1154 & -1.09 & 0.2770 \\
\hline V-D value & 2.5738 & 0.5487 & 4.69 & $<0.0001$ \\
\hline
\end{tabular}


TABLE 4 | Multivariate Logistic regression between recurrence and multiple factors.

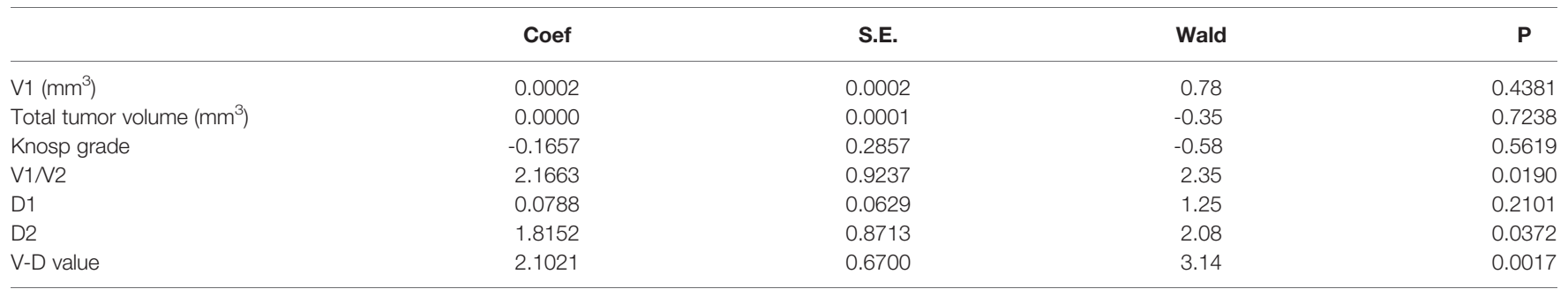

compressed tissue surrounding the macroadenoma $(1,5)$. Therefore, the treatment and prognostic factors for NFPAs should be taken seriously. Our study has provided a brief and effective method for predicting the probability of recurrence in patients with NFPAs preoperatively, using some radiological indices.

Some studies have used molecular markers for the prediction of recurrence (15). However, pathology results can only be obtained after surgery, and few factors can predict recurrence before surgery. Predicting recurrence before surgery is essential not only for reducing patients' distress but also for providing individual advice for their treatment. If a high potential of recurrence in a patient can be detected before surgery, a more aggressive surgery plan could be drawn up, and the follow-up plan adhered to more diligently. We found that a higher V-D value was positively related to tumor recurrence after surgery, showing good efficacy in the prediction of residual tumors (Figures 3, 4). The V-D value can be obtained from preoperative radiological data and used in the preoperative discussion when seeking patient consent. For those with higher V-D values, surgeons can devise more radical strategies, such as resection of the internal wall of the cavernous sinus or extended transsphenoidal surgery. Intraoperative MRI detection can also be performed for those with higher $\mathrm{V}$-D values to provide the greatest chance for achieving total resection.

In our study, patients with a V-D value higher than 1.53 were predicted to undergo relapse much earlier than those with a lower V-D value. On the one hand, this value represents a quantification of the difficulty of total resection during transsphenoidal surgery. Patients with residual tumors have a greater risk of progression postoperatively according to previous studies $(5,15-18)$. The relapse rate was higher after 10 years of follow-up than after only 5 years of follow-up in patients with a residual tumor. There was an interesting case in our study that showed the potential of quantifying surgical difficulty using the $\mathrm{V}-\mathrm{D}$ value. A patient with a large pituitary adenoma (TV: $31626.76 \mathrm{~mm}^{3}, \mathrm{~V} 2: 1604.80 \mathrm{~mm}^{3}$, V1: $30021.96 \mathrm{~mm}^{3}$ ) only had a V-D value of 1.11 because the principal part of the tumor was located in the intra- and infrasellar regions, which were easily totally resected using endoscopes. Therefore, according to our results, follow-up should be performed for an
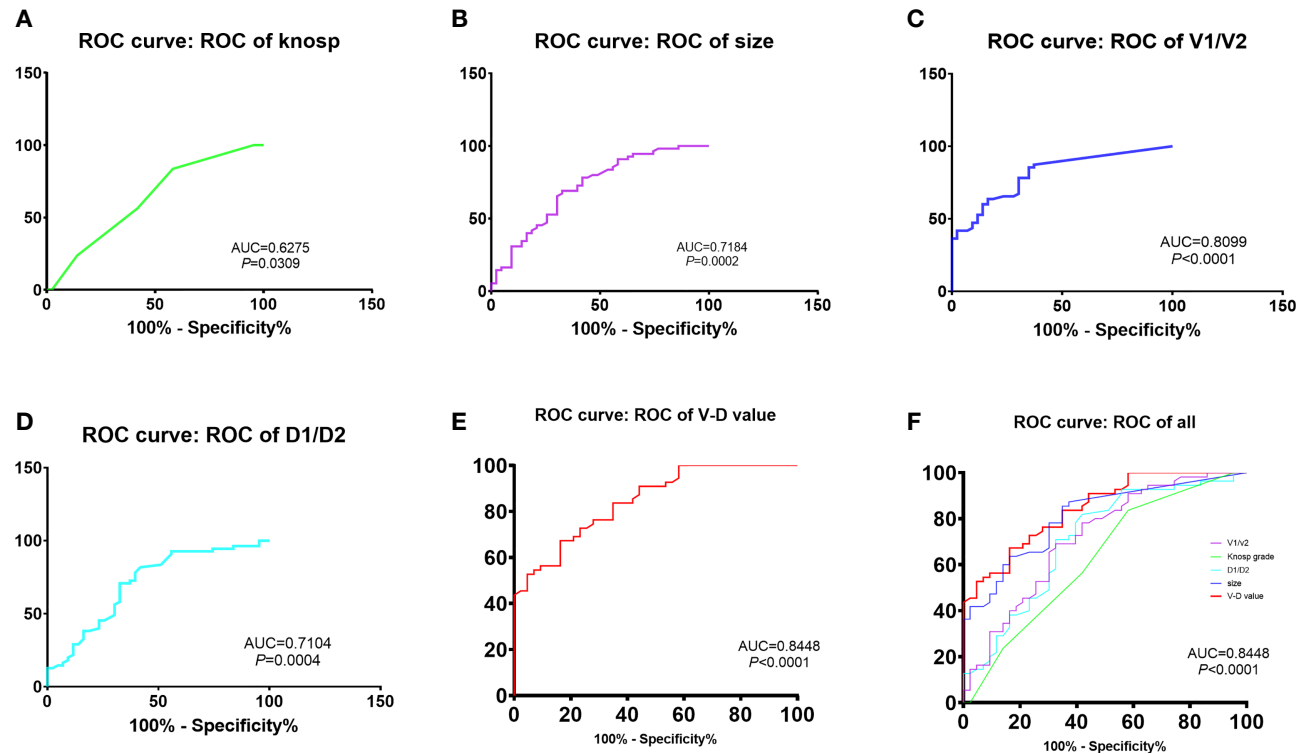

FIGURE 1 | Comparison of recurrent time divided by the cut-off value of V-D: The predictive efficacy of knosp grades (A), tumor size (B), ratio of V1 and V2 (C), ratio of D1 and D2 (D) and $V$-D value (E). The $V$-D value has shown the best efficacy in predicting recurrence in NFPAs (AUC $=84.48 \%$, sensitivity $=0.837$, specificity $=0.673$, cut-off value $=1.53$. 


$$
\text { Strata }=\mathrm{VD}=0-\mathrm{VD}=1
$$

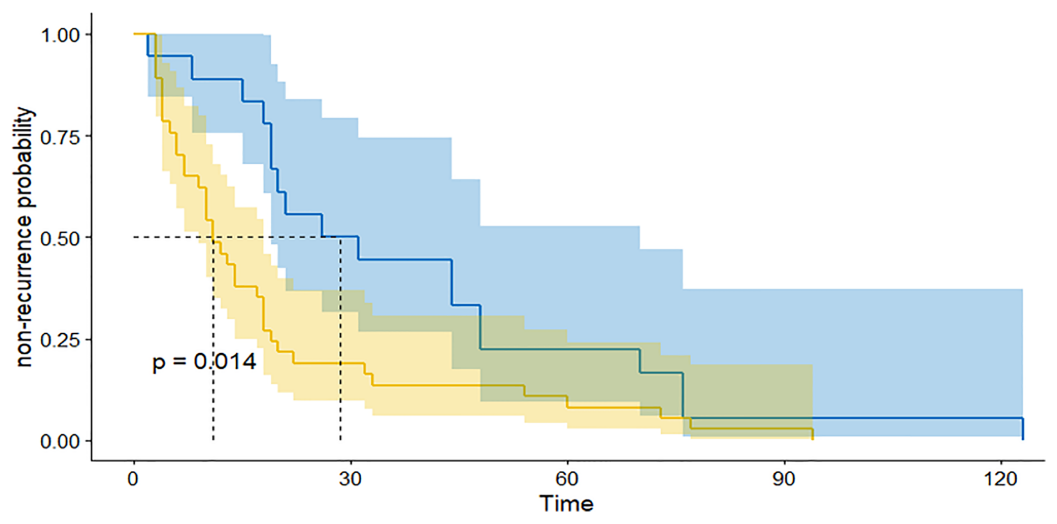

FIGURE 2 | ROC for the prediction of recurrence by several indexes: The recurrent time of patients with high V-D value (yellow line) was significantly shorter than patients with low V-D value (blue line).

indefinite period and more intensively for those with tumors with a high $V$-D value.

This value may reflect the growth pattern of tumors with higher invasiveness, which should be verified by further molecular studies. Ki-67 is a classical index for the description of tumor proliferation in pituitary adenomas $(19,20)$. However, current estimations of Ki-67 are mostly based on visual detection under a microscope, which lacks objectivity. In our study, the V-D value did not correlate with the level of Ki-67, suggesting that invasiveness or proliferation may not directly correlate with recurrence probability. As the examination of transcription factors was incomplete in our patients, we did not study the relationship. Further studies on molecular features such as the expression of pit-1, t-pit and p53, as well as the V-D value are necessary to provide deeper insights into the prediction methods.

With the development of artificial intelligence and data science, many studies have used radiomics methods or machine learning to predict the recurrence of brain tumors $(18,21,22)$. However, models built by those methods lack universality between different study centers due to the heterogeneity of data collection, which is the key to the accuracy of model building. Although some models may show higher predictive value, they need complicated post-
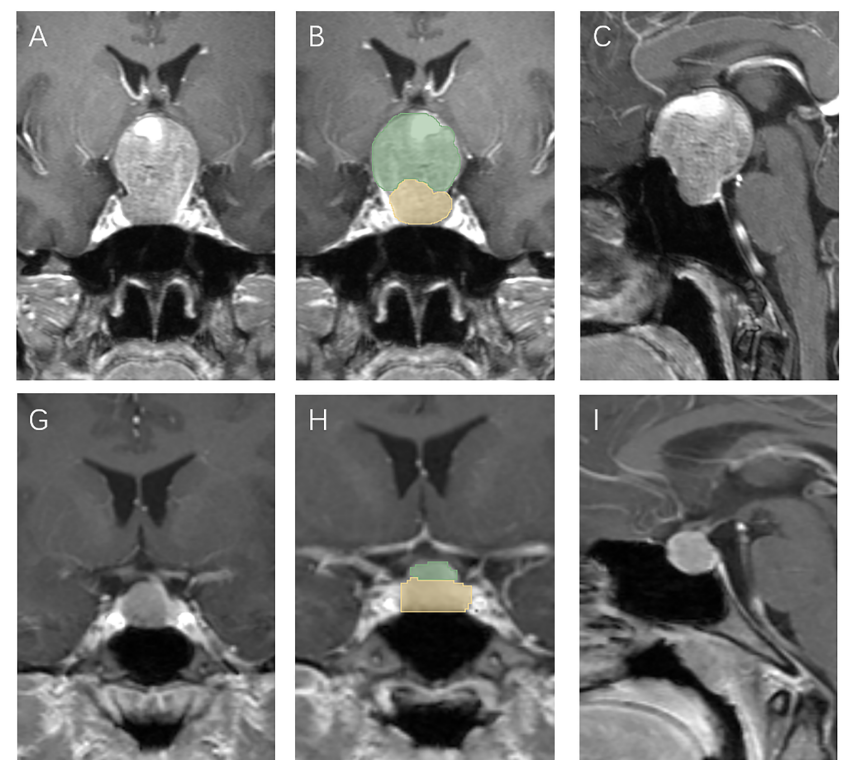
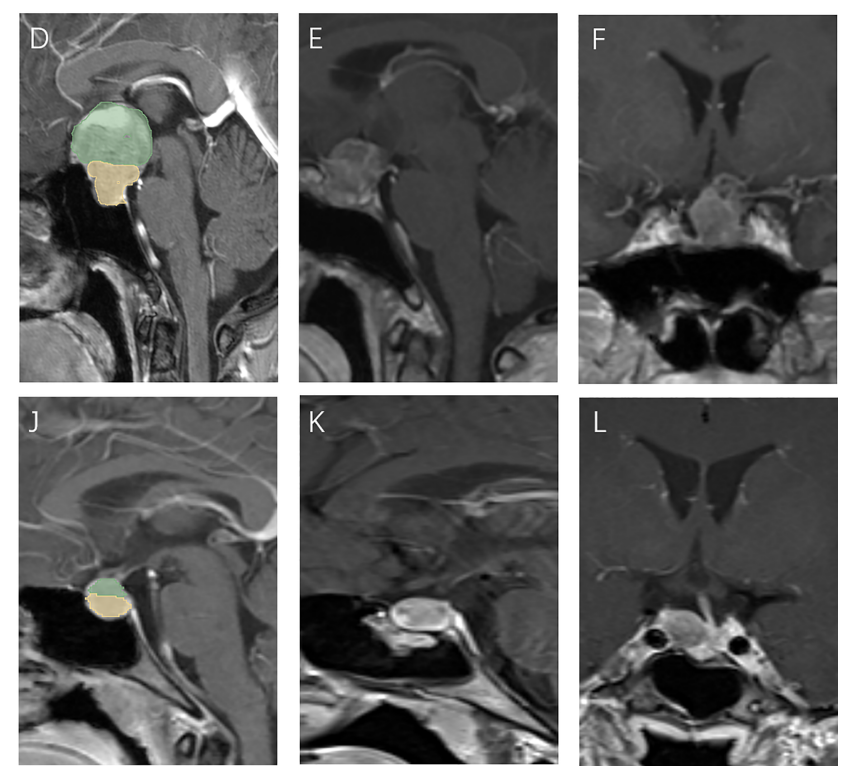

FIGURE 3 | Patients with high V-D value: A 51 year old female, TV: $13699.48 \mathrm{~mm}^{3}$, V2: $1620.19 \mathrm{~mm}^{3}$, V1:12079.29 mm³ , V-D: 8.64 (A-D), relapse 13 months after surgery (E, F); a 30 year old female, TV: $470.46 \mathrm{~mm}^{3}$, V2: $186.48 \mathrm{~mm}^{3}$, V1:283.98 mm³, V-D: 2.56 (G-J), relapse 94 months after surgery (K, L). 

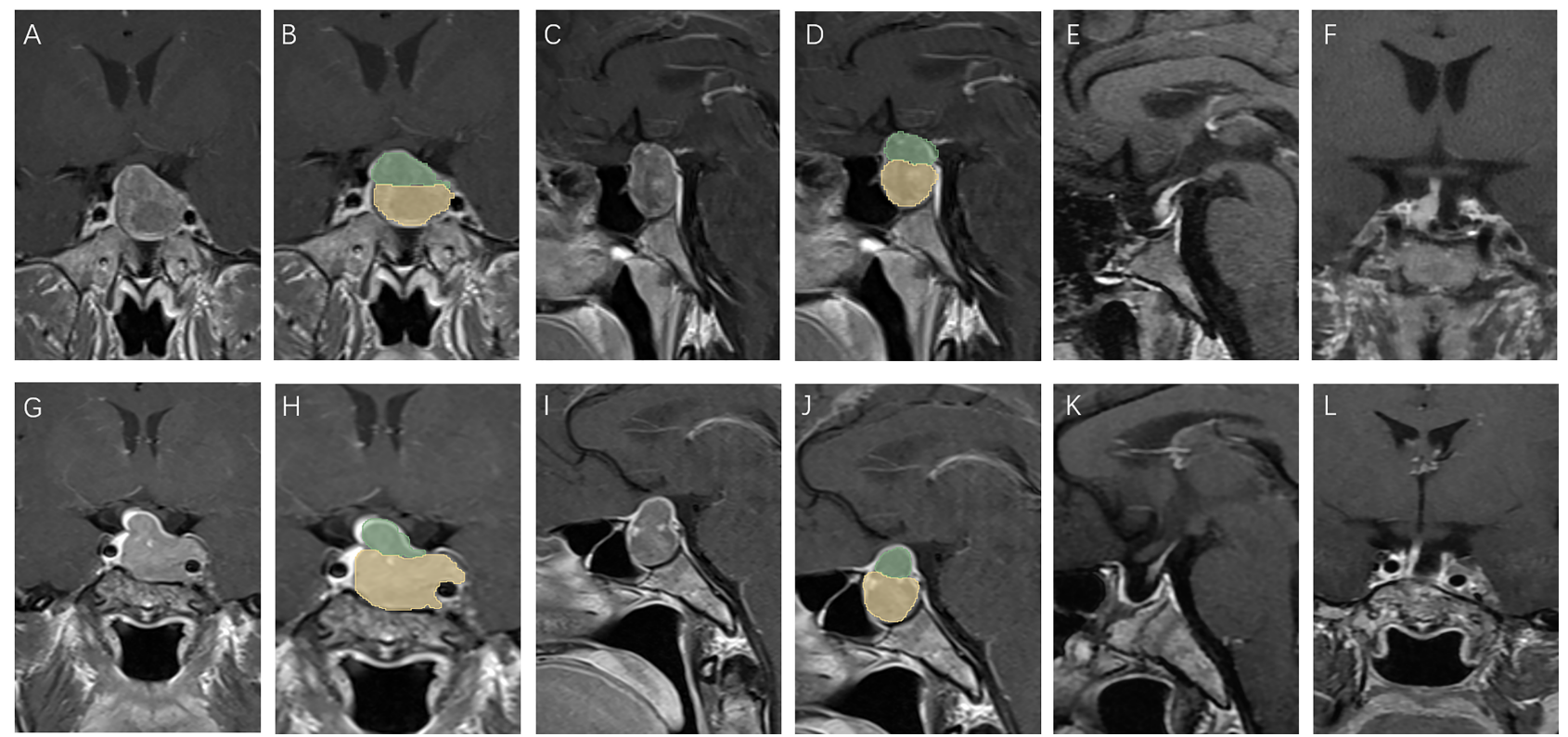

FIGURE 4 | Patients with low V-D value: A 39 year old male, TV: 5781.19 mm³ $^{3}$, V2: $4376.40 \mathrm{~mm}^{3}$, V1:1404.79 mm³ , V-D: 1.19 (A-D), no relapse in 7 years of follow-up (E, F); a 43 year old female, TV: $5888.45 \mathrm{~mm}^{3}$, V2: $5188.17 \mathrm{~mm}^{3}$, V1:700.28 mm³, V-D: 1.31 (G-J), no relapse in 7 years of follow-up (K, L).

processing of radiological and clinical data. The V-D value provided in our study is rapidly calculated not only on some open-source software but also on many hospital platforms and even PACS systems in many centers. Different from the variability of radiomics features, the tumor volume and diameter will not change regardless of where the data are analyzed.

\section{Limitations}

The first limitation of our study was the small sample size and lack of external validation. Larger and multicenter datasets should be analyzed further to verify our results in the prediction of recurrence in NFPAs. Second, as the patients in our center did not routinely undergo CTA or MRA before surgery, the carotid distance could only be roughly detected on common MRI for convenience. The carotid artery could be reconstructed using CTA or MRA preoperatively, to increase the accuracy of the D2 value in further studies. Third, most patients did not undergo detection of transcription factors, which could be used for further verification of the utility of the V-D value. Lastly, an automated algorithm for tumor segmentation will be needed for the accurate calculation of tumor volume in further studies.

\section{CONCLUSION}

In conclusion, we discovered that there was a positive relationship between the V-D value and tumor recurrence of NFPAs. Patients with higher $\mathrm{V}$-D values showed a higher probability of relapse and shorter recurrence time. Those with a higher $\mathrm{V}-\mathrm{D}$ value should be given more attention in terms of surgery and follow-up.

\section{DATA AVAILABILITY STATEMENT}

The original contributions presented in the study are included in the article/supplementary material. Further inquiries can be directed to the corresponding authors.

\section{ETHICS STATEMENT}

The studies involving human participants were reviewed and approved by Sun Yat-Sen University. The ethics committee waived the requirement of written informed consent for participation.

\section{AUTHOR CONTRIBUTIONS}

Conception and design: WC, MW, and CD. Drafting the article: WC and MW. Acquisition of data: WC and HW. Analysis and Interpretation of data: all authors. Critically revising the article: SY and HW. Approved the final version of manuscript on behalf of all authors: HW. Statistical analysis: WC and MW. Graphic visualization: WC and MW. Clinical diagnosis and radiological identification: WC, $\mathrm{ZW}, \mathrm{BH}$, and ZM. Administrative/technical/material support: WC, ZM, YZ, and HW. Study supervision: HW and YZ. All authors contributed to the article and approved the submitted version.

\section{FUNDING}

The study was funded by Clinical Research Project of The East Division (the First Affiliated Hospital, Sun Yat-sen University, 2019004), Guangdong Natural Science Foundation (2018A0303130333), and The Chinese Postdoctoral Science Foundation (2019M663271). 


\section{REFERENCES}

1. Kasper G, Samuel N, Alkins R, Khan OH. Practice Patterns in the Management of Recurrent and Residual Non-Functioning Pituitary Adenomas: Results From a Canada-Wide Survey. eNeurologicalSci (2021) 22:100317. doi: 10.1016/j.ensci.2021.100317

2. Lee MH, Hur KY, Hong SD, Seol HJ, Choi JW, Lee JI, et al. Early Hormonal Recovery Following Endoscopic Transsphenoidal Surgery for Silent Non-Functioning Pituitary Adenomas With Hormone Dysfunction. J neuro-oncol (2021) 153:343-50. doi: 10.1007/s11060-02103774-y

3. Lelotte J, Mourin A, Fomekong E, Michotte A, Raftopoulos C, Maiter D. Both Invasiveness and Proliferation Criteria Predict Recurrence of NonFunctioning Pituitary Macroadenomas After Surgery: A Retrospective Analysis of a Monocentric Cohort of 120 Patients. Eur J Endocrinol (2018) 178:237-46. doi: 10.1530/eje-17-0965

4. Abdelmannan D, Aron DC. Incidentally Discovered Pituitary Masses: Pituitary Incidentalomas. Expert Rev Endocrinol Metab (2010) 5:253-64. doi: $10.1586 /$ eem. 09.68

5. Iglesias P, Arcano K, Triviño V, Guerrero-Pérez F, Rodríguez Berrocal V, Vior C, et al. Giant Non-Functioning Pituitary Adenoma: Clinical Characteristics and Therapeutic Outcomes. Exp Clin Endocrinol Diabetes: Off J German Soc Endocrinol German Diabetes Assoc (2021) 129:309-13. doi: 10.1055/a-1017-3288

6. Bastos RV, Silva CM, Tagliarini JV, Zanini MA, Romero FR, Boguszewski CL, et al. Endoscopic Versus Microscopic Transsphenoidal Surgery in the Treatment of Pituitary Tumors: Systematic Review and Meta-Analysis of Randomized and Non-Randomized Controlled Trials. Arch Endocrinol Metab (2016) 60:411-9. doi: 10.1590/2359-3997000000204

7. Cortet-Rudelli C, Bonneville JF, Borson-Chazot F, Clavier L, Coche Dequéant B, Desailloud R, et al. Post-Surgical Management of Non-Functioning Pituitary Adenoma. Annales d'endocrinologie (2015) 76:228-38. doi: 10.1016/ j.ando.2015.04.003

8. Roelfsema F, Biermasz NR, Pereira AM. Clinical Factors Involved in the Recurrence of Pituitary Adenomas After Surgical Remission: A Structured Review and Meta-Analysis. Pituitary (2012) 15:71-83. doi: 10.1007/s11102011-0347-7

9. Abdelmaksoud A, Fu P, Alwalid O, Elazab A, Zalloom A, Xiang W, et al. Degrees of Diaphragma Sellae Descent During Transsphenoidal Pituitary Adenoma Resection: Predictive Factors and Effect on Outcome. Curr Med Sci (2018) 38:888-93. doi: 10.1007/s11596-018-1958-2

10. Lv L, Yin S, Zhou P, Hu Y, Chen C, Ma W, et al. Clinical and Pathologic Characteristics Predicted the Postoperative Recurrence and Progression of Pituitary Adenoma: A Retrospective Study With 10 Years Follow-Up. World Neurosurgery (2018) 118:e428-35. doi: 10.1016/ j.wneu.2018.06.210

11. Staartjes VE, Serra C, Muscas G, Maldaner N, Akeret K, van Niftrik CHB, et al. Utility of Deep Neural Networks in Predicting Gross-Total Resection After Transsphenoidal Surgery for Pituitary Adenoma: A Pilot Study. Neurosurgical Focus (2018) 45:E12. doi: 10.3171/2018.8.Focus18243

12. Lyu W, Fei X, Chen C, Tang Y. Nomogram Predictive Model of PostOperative Recurrence in Non-Functioning Pituitary Adenoma. Gland Surg (2021) 10:807-15. doi: 10.21037/gs-21-47
13. Yan X, Chen X, Ge H, Zhu S, Lin Y, Kang D, et al. The Change in Distance Between Bilateral Internal Carotid Arteries in Acromegaly and Its Risk Factors. Front Endocrinol (2020) 11:429. doi: 10.3389/fendo.2020.00429

14. Egger J, Kapur T, Nimsky C, Kikinis R. Pituitary Adenoma Volumetry With 3D Slicer. PloS One (2012) 7:e51788. doi: 10.1371/journal.pone.0051788

15. Machado LF, Elias PCL, Moreira AC, Dos Santos AC, Murta Junior LO. MRI Radiomics for the Prediction of Recurrence in Patients With Clinically NonFunctioning Pituitary Macroadenomas. Comput Biol Med (2020) 124:103966. doi: 10.1016/j.compbiomed.2020.103966

16. Pérez-López C, Palpán AJ, Saez-Alegre M, Zamarrón A, Alfonso C, Álvarez-Escola C, et al. Volumetric Study of Nonfunctioning Pituitary Adenomas: Predictors of Gross Total Resection. World Neurosurgery (2020) 147:e206-14. doi: 10.1016/j.wneu.2020.12.020

17. Yavropoulou MP, Tsoli M, Barkas K, Kaltsas G, Grossman A. The Natural History and Treatment of Non-Functioning Pituitary Adenomas (NonFunctioning Pitnets). Endocrine-Related Cancer (2020) 27:R375-r390. doi: 10.1530/erc-20-0136

18. Heng L, Liu X, Jia D, Guo W, Zhang S, Gao G, et al. Preoperative Prediction of Granulation Pattern Subtypes in GH-Secreting Pituitary Adenomas. Clin Endocrinol (2021) 95:134-42. doi: 10.1111/cen.14465

19. Conficoni A, et al. Biomarkers of Pituitary Macroadenomas Aggressive Behaviour: A Conventional MRI and DWI 3T Study. Br J Radiol (2020) 93:20200321. doi: 10.1259/bjr.20200321

20. Cheng S, Guo J, Zhang Y, Li Z, Li C. Identification of a Multidimensional Transcriptome Signature Predicting Tumor Regrowth of Clinically Non-Functioning Pituitary Adenoma. Int J Oncol (2020) 57:804-12. doi: $10.3892 /$ ijo. 2020.5087

21. Vuong HG, Nguyen TPX, Pham N, Dunn IF. Risk Factors for Tumor Recurrence and Progression of Spindle Cell Oncocytoma of the Pituitary Gland: A Systematic Review and Pooled Analysis. Pituitary (2020) 24(3):42937. doi: 10.1007/s11102-020-01110-7

22. Vivancos Sánchez C, et al. Role of Pituitary Stalk and Gland Radiological Status on Endocrine Function and Outcome After Endoscopic Transsphenoidal Surgery for Non-Functioning Pituitary Adenomas. Endocrine (2021) 73:41523. doi: $10.1007 / \mathrm{s} 12020-021-02726-\mathrm{w}$

Conflict of Interest: The authors declare that the research was conducted in the absence of any commercial or financial relationships that could be construed as a potential conflict of interest.

Publisher's Note: All claims expressed in this article are solely those of the authors and do not necessarily represent those of their affiliated organizations, or those of the publisher, the editors and the reviewers. Any product that may be evaluated in this article, or claim that may be made by its manufacturer, is not guaranteed or endorsed by the publisher.

Copyright (ङ 2021 Chen, Wang, Duan, Yao, Jiao, Wang, Hu, Mao, Zhu and Wang. This is an open-access article distributed under the terms of the Creative Commons Attribution License (CC BY). The use, distribution or reproduction in other forums is permitted, provided the original author(s) and the copyright owner(s) are credited and that the original publication in this journal is cited, in accordance with accepted academic practice. No use, distribution or reproduction is permitted which does not comply with these terms. 